\title{
Therapeutic Effects of Laser on Partial Osteotomy in the Rat Model of Hypothyroidism
}

\author{
Niloofar Sefati ${ }^{1}$, Hojjat-Allah Abbaszadeh ${ }^{2,1^{*}}$, Fatemeh Fadaei Fathabady ${ }^{3,1}$, Mohammad-Amin Abdollahifar ${ }^{1}$, \\ Abdollah Amini' ${ }^{1}$, Ali Noori-Zadeh ${ }^{4}$, Shahram Darabi ${ }^{5}$, Mohsen Norouzian ${ }^{3,1^{*}}$ \\ ${ }^{1}$ Department of Biology and Anatomical Sciences, School of Medicine, Shahid Beheshti University of Medical Sciences, \\ Tehran, Iran \\ ${ }^{2}$ Hearing Disorders Research Center, Loghman Hakim Hospital, Shahid Beheshti University of Medical Sciences, Tehran, \\ Iran \\ ${ }^{3}$ Laser Application in Medical Sciences Research Center, Shahid Beheshti University of Medical Sciences, Tehran, Iran \\ ${ }^{4}$ Department of Clinical Biochemistry, Faculty of Paramedicine, Ilam University of Medical Sciences, Ilam, Iran \\ ${ }^{5}$ Cellular and Molecular Research Center, Qazvin University of Medical Science, Qazvin, Iran
}

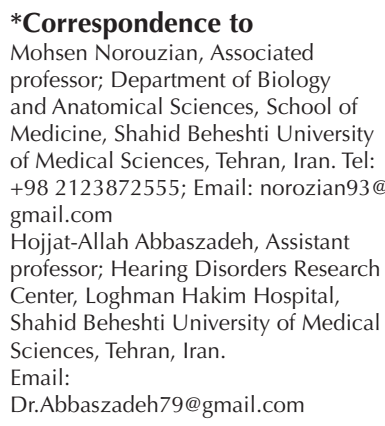

Published online 20 March 2018

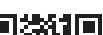

\begin{abstract}
Introduction: Several experimental studies have displayed positive result for laser radiation on stimulating bone regeneration in recent years. The purpose of this experimental study was to determine low-level laser (LLL) effects on partial bone defects in hypothyroidism male rat.

Methods: Forty male Wistar rats were randomly distributed as below groups: hypothyroidism + laser (Hypo + laser), hypothyroidism (Hypo), and control. Four weeks after surgery, the tibia bone was removed. Biomechanical and histological examinations were performed immediately.

Results: Our results showed significant reduction in the absorption of energy, resistance in bending deformation (bending stiffness), maximum force, high stress load, trabecular bone volume, and number of osteocytes, osteoblasts and osteoclasts in the osteotomy site in hypothyroidism rats compared to hypothyroidism + laser group $(P<0.05)$.

Conclusion: The results indicated that using laser may improve fracture regeneration and it may accelerate bone healing in hypothyroidism rat.

Keywords: Hypothyroidism; Low-level laser; Partial osteotomy.
\end{abstract}

\section{Introduction}

Thyroid hormones have an important role in our development and in biochemical homeostasis of the different tissues in human body such as nerve system and skeletal system. Triiodothyronine (T3) and tetraiodothyronine (T4) are bone evolution regulators and essential for bone growth and nerve development. Reduced thyroid hormones or their receptors causes a variety of disorders in body metabolism. ${ }^{1-3}$ Hypothyroidism disrupts bone formation and reabsorption which impairs bone regeneration. ${ }^{4}$ The most common treatment for hypothyroidism is hormone therapy, and most patients require this process throughout their lives. ${ }^{5}$ Treating these patients with levothyroxine can increase the risk of osteoporosis and fracture. ${ }^{6}$ Due to hypothyroidism-induced abnormalities in the development of the skeletal system, the presence of a new bone to replace or maintain the function of the affected bones is a critical need in clinical aspects and in this respect, one of the therapies for bone repair is laser therapy. Recently, there has been a dramatic change in the clinical applications of laser. In various studies, the effects of low-level laser (LLL) radiation on various tissues have been reported, especially on angiogenesis and accelerated bone remodeling. ${ }^{7,8}$ Studies have shown that LLL radiation can have excitatory effects on bone tissue. Studies have shown that laser radiation is absorbed by the mitochondrial electron chain, which increases the production of ATP, oxygen, and protein synthesis, which ultimately lead to mitosis and cell proliferation. ${ }^{9,10}$ On the other hand, LLL can increase reactive oxygen species (ROS) in various types of cells, and evidence suggests that low levels of ROS activate MAPK (mitogenactivated protein kinase) signaling pathways that control cell proliferation and differentiation processes, while high laser doses cause excessive ROS production and disruption of the physiological function of the cell. ${ }^{11,12}$ Laser can induce osteoblasts and bone formation. Also, laser can increase functioning and differentiation of osteoclasts. By increasing bone cells functioning, bone regeneration 
increases and bone repair is accelerated. ${ }^{13,14}$ Considering the effect of the thyroid hormones on bone formation, failure to fully repair fractures and the undesirable effects of conventional treatments, the present study examined the effects of LLL radiation in treating bone fracture in hypothyroid rats.

\section{Methods \\ Animal \\ In this experimental study, 40 rats (adult Wistar males) weighing 190-220 g were used. The animals were kept in the animal lab for 2 weeks to adapt to new conditions. During the study, the rats had free access to water and standard feed. Lab conditions were controlled at a temperature of about $22^{\circ} \mathrm{C}$ and a 12 -hour brightness cycle. The rats relaxed by injecting an anesthetic drug during laser irradiation, and during the screening. Methimazole powder was used to induce hypothyroidism. The methimazole powder was given to the rats for 4 weeks (28 days) in edible water (distilled water). After 28 days, blood was taken again from the eye cornea of the mouse, and after an analysis of the amount of hormones and with a lack of thyroid function, we performed further analyses.}

\section{Thyroid Hormones}

Regarding the induction of hypothyroidism by methimazole, T4 and T3 hormones were expected to be reduced in the peripheral circulation. Blood samples were taken from the eye cortex in days 1 and 28 and serum T4, T3 levels were measured by ELISA immunoassay.

\section{Partial Osteotomy}

The rats were anesthetized by ketamine and diazepam injections. After cutting the skin and pushing the muscles, a minor lesion in the tibia bone of the two sides was created with a fine-toothed circular micro-saw at the midpoint of the tibia trunk with a diameter of $1.5 \mathrm{~mm}$. The muscle was sewn by catgut 04 , and the skin was sewn by the threaded nylon reversed cutting 04. Ceftriaxone antibiotic was injected intramuscularly at the time of surgery, 24 and 48 hours after.

\section{Research Groups}

After induction of bone defects, the rats were distributed into three groups, including: control, hypothyroidism (Hypo) and hypothyroidism + laser (Hypo + laser). In the control group, the animals were developed without bilateral bone defect, without methimazole and then were kept for one month without any intervention. In the laser group, after receiving methimazole, inducing hypothyroidism, and creating bone defects, a LLL was applied on up to 3 points with the following specifications (at the site of bone defect, $0.5 \mathrm{~cm}$ below and $0.5 \mathrm{~cm}$ above the defect).

Laser radiation was immediately applied after defect induction and it lasted for four weeks. Laser radiation was performed once, every three days. For each point, an infrared laser $890 \mathrm{~nm}$ wavelength, $80 \mathrm{~Hz}$ of frequency for 20 minutes at a dose of $1.5 \mathrm{~J} / \mathrm{cm}^{2}$ was irradiated (Table 1). Four weeks after the surgery, the studied animals were euthanized with anesthetic injections and the right and left tibia bones were sampled. Ultimately, the right tibia was subjected to biomechanical and the left tibia was subjected to histological studies. The right tibia bone was completely removed from the body, the muscles and fibula attached to it were removed. Then, the tibia was placed in a piece of gas impregnated with $0.9 \%$ saline solution and transferred to the freezer at minus $20^{\circ} \mathrm{C}$. After de-freezing the bones to room temperature, the two ends of the bone were placed on the two edges of the support holders of the material strength test (Zwick, Germany). The distance between the two supporting points was $20 \mathrm{~mm}$. The orientation of all samples was considered the same. The specification, thickness and width of the sample area were given to the device at the site of the bone defect. The movable base applied a force at a speed of $0.08 \mathrm{~mm} / \mathrm{s}$ to the midpoint of the bone. The load-deformation curve was pulled by a computer connected to the machine. The elastic stiffness, absorbed energy, maximum force and maximum stress were provided by the computer. The elasticity of the gradient refers to the linear gradient of the load-deformation curve, i.e. the ratio of load to deformation in the elastic curvature. The energy absorbed was defined as the amount of energy absorbed by the bone specimen to the breaking point. The force refers to maximum force that can break the bone.

\section{Biomechanical Test}

The experimental rats in each group were sacrificed four weeks after the surgery, and then, the right tibia bones were separated from other additional tissues and weighed. For the purpose of biomechanical studies, five tibia bones per groups were tested. Three point bending assessment of the collected sample bones were performed as previously reported (Zwick, Roell Group, Z 2.5 H 15WN, Ulm, Germany). In the tensiometery device, the entire bones were located in similar orientation. Two points of loading part with $19 \mathrm{~mm}$ in length were applied to the sustenance of two bones; a press pate was then started to squash the midpoint of bones until breakage happened.

Table 1. The Characteristics of the Laser Used

\begin{tabular}{lc}
\hline Parameters & Dose and Unit \\
\hline Peak power output & $80 \mathrm{~W}$ \\
Average power & $1.15 \mathrm{~mW}$ \\
Power density & $1.15 \mathrm{~W} / \mathrm{cm}^{2}$ \\
Wavelength & $890 \mathrm{~nm}$ \\
Pulse frequency & $80 \mathrm{~Hz}$ \\
Pulsed duration & $180 \mathrm{~ns}$ \\
Each point duration of exposure & $1300 \mathrm{~s}$ \\
Energy density & $1.5 \mathrm{~J} / \mathrm{cm}^{2}$ \\
\hline
\end{tabular}


The speed of loading parts was about $0.08 \mathrm{~mm} / \mathrm{s}$ during test. The data from tensiometery were collected from the device, which was obtained from the curve of loaddeformation. In this study the parameters including; the amount of energy absorption $(\mathrm{N} / \mathrm{mm})$, bending stiffness $(\mathrm{N} / \mathrm{mm})$, maximum force $(\mathrm{N})$, and high load stress $(\mathrm{N} /$ $\mathrm{mm}^{2}$ ) were evaluated. Bending stiffness refers to the slant on the linear part in the load-deformation curve. In this case energy refers to total energy absorbed by each bone until it break. Maximum force is defined as force used to breakdown the bones. The high load stress was obtained by dividing $\mathrm{N}$ in the bone area $\left(\mathrm{mm}^{2}\right)$ in the osteotomy region. ${ }^{1}$

\section{Tissue Preparation}

Histological evaluation was performed after surgery. The bone sample (right tibia) was removed and fixed in buffer formalin (10\%) for 48 hours and then decalcified in 10\% nitric acid for 2 weeks. The bone defected areas were cut longitudinally by microtome (10 $\mu \mathrm{m}$ thick). The tissue samples were stained by hematoxylin and eosin (H\&E).

\section{Stereological Evaluation}

Bone Volume

The volumes of the trabecular bone, bone marrow, cortical bone, and fibrous tissue were estimated with the Cavaliers method. ${ }^{15}$

\section{Bone Cells Number}

The cell density and total numbers of osteoblast, osteoclast, and osteocyte were analyzed by the optical dissector method. Sections were measured with the optical dissector methods. ${ }^{16}$

\section{Statistical Analysis Method}

The determination of the normal distribution of data was done by Kruskal-Wallis test. To test the statistical difference between the groups studied in different parts of the study, we used one-way ANOVA and LSD in the case of normal data distribution, Kruskal-Wallis test and Mann-Whitney test in the case of failure to have a natural distribution of data. A significant level of $P<0.05$ was considered for ANOVA, LSD and $P<0.01$ for Mann Whitney test.

\section{Results}

Thyroid Hormones Levels

The results show that the induction of hypothyroidism by means of methimazole reduced $\mathrm{T} 3$ and $\mathrm{T} 4$ hormones in the peripheral blood (Table 2).

\section{Maximum Force (N)}

As shown in Figure 1, the group (hypo+ laser) exposed to a wavelength of $890 \mathrm{~nm}$ and a frequency of $80 \mathrm{~Hz}$ for 20 minutes for each point at a dose of $1.5 \mathrm{~J} / \mathrm{cm}^{2}$, had no significant increase in maximum force compared with
Table 2. Thyroid Hormones in the Control and Hypothyroidism Groups

\begin{tabular}{lcc}
\hline Hypothyroidism Group & Control Group & Thyroid Hormones \\
\hline $21.28 \pm 8.41 \mathrm{ng} / \mathrm{dL}$ & $98.12 \pm 1.43 \mathrm{ng} / \mathrm{dL}$ & $\mathrm{T} 3$ \\
$1.53 \pm 0.18 \mu \mathrm{g} / \mathrm{dL}$ & $4.02 \pm 1.31 \mu \mathrm{g} / \mathrm{dL}$ & $\mathrm{T} 4$ \\
\hline
\end{tabular}

the Hypo groups. There was no significant alteration in maximum force in the Hypo group. Laser groups in comparison with the control groups (LSD test; $P=0.000$ ) in Figure 2.

High Stress Load $\left(\mathrm{N} / \mathrm{mm}^{2}\right)$

As shown in Figure 2, the High stress load was lower in the hypo groups than in the control and Hypo + laser groups. However, Hypo + laser indicated a significant growth in the high stress load in comparison with the Hypo group $(P=0.000$; Figure 2).

\section{Energy Absorption (N/mm)}

Our study revealed a decreased energy absorption in Hypo and control groups in comparison with Hypo + laser group. Energy absorption was obviously increased in hypo + laser group in comparison with Hypo groups

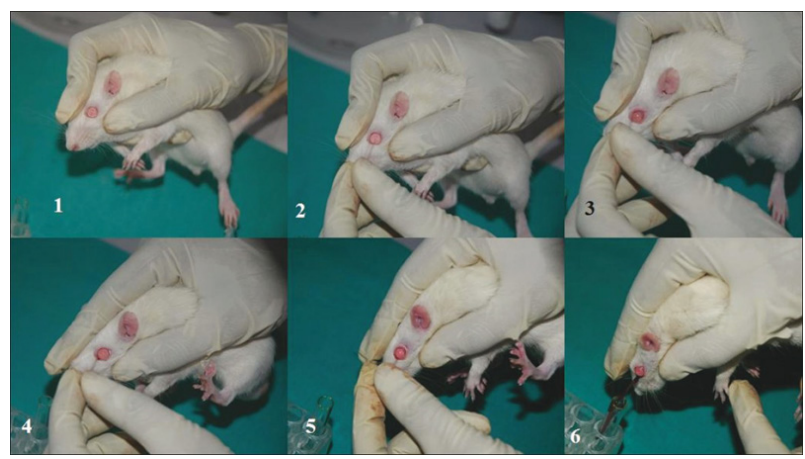

Figure 1. Blood Sampling From the Eye of the Rat's to Evaluate Thyroid Hormone Synthesis.

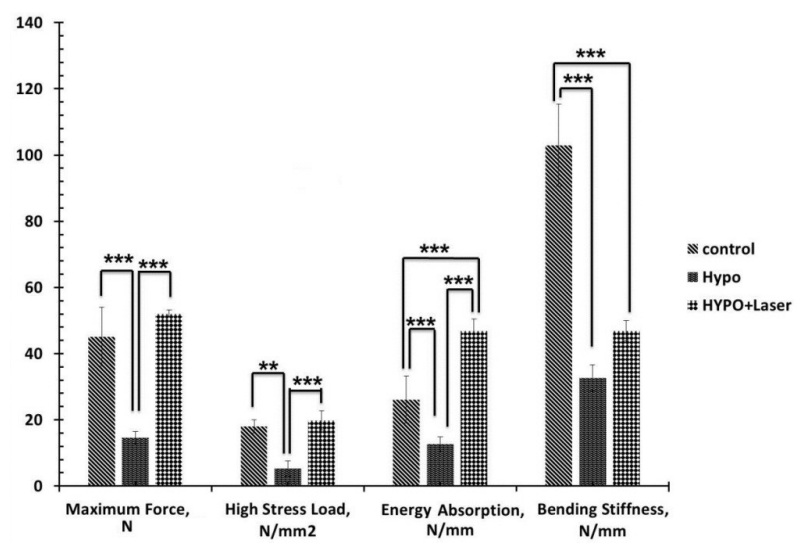

Figure 2. Mean \pm SD of Biomechanical Properties in the 3 Study Groups. 
(LSD test; $P=0.000$; Figure 2).

Bending Stiffness (N/mm)

The study of the data obtained from bending stiffness analysis showed that the use of laser causes a dramatically increase in maximum force in comparison with other groups. The maximum force level was higher in the control group when compared with Hypo and Hypo + laser groups (LSD test; $P=0.000, P=0.000$; Figure 2).

Analysis of Stereological Parameters

ANOVA analysis was performed for data derived from stereological parameters in different groups of research. The results for bone density are shown in Figures 3-8. The results of comparing different groups are as follows: There is a significant difference in the volume of bone marrow between the laser group and the control group $(P<0.01)$. There was a significant difference between the groups in terms of cortical tissue volume $(P<0.01)$. There was a significant difference in the volume of trabecular tissue between the hypothyroid group and the control group $(P<0.01)$. There was a significant difference between the hypothyroid group and the laser group in terms of trabecular tissue volume.

For each group, the number of bone cells has been shown in Figures 6-9. The results of comparing different groups are as follows: There was a significant difference between

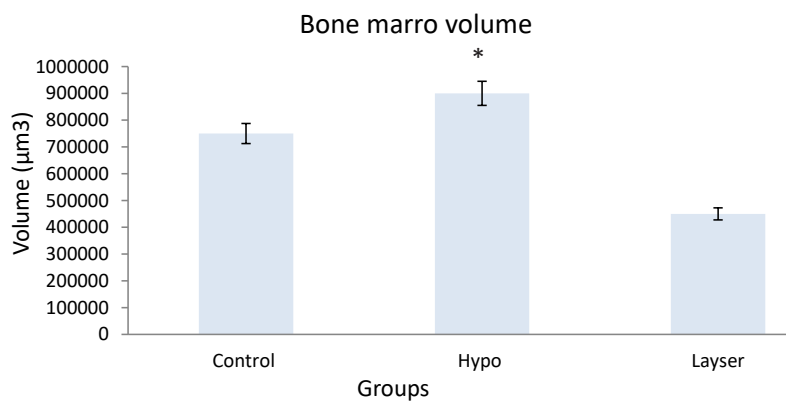

Figure 3. Mean \pm SEM of Bone Marrow Volume (BMV) in Different Groups.

* shows the significant difference between Hypo group and the other groups $(P<0.01)$

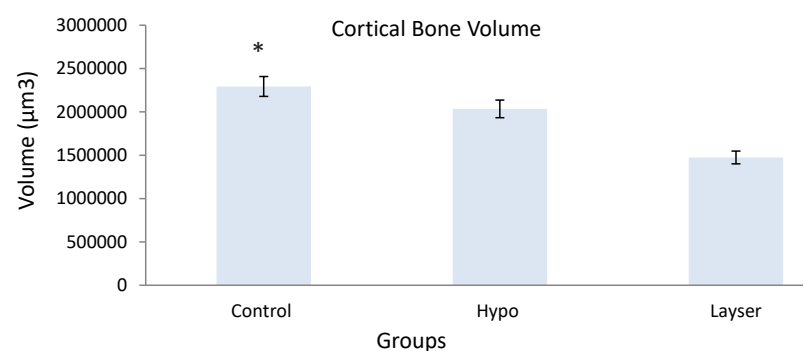

Figure 4. Mean \pm SEM of Cortical Bone Volume in Different Groups. * shows the significant difference between Hypo group and the other groups $(P<0.01)$ the number of osteocytes for the hypothyroid and control groups $(P<0.05)$. There was a significant difference between the number of osteocytes for the hypothyroid and laser groups $(P<0.01)$. There was a significant difference in the number of osteoblasts between the hypothyroid group and the control group $(P<0.05)$. There was a significant difference between the number of osteoblasts of the hypothyroid and the laser groups $(P<0.01)$. There was a significant difference in the number of osteoclasts between the hypothyroid group and the control group $(P<0.05)$. There was a significant difference between the

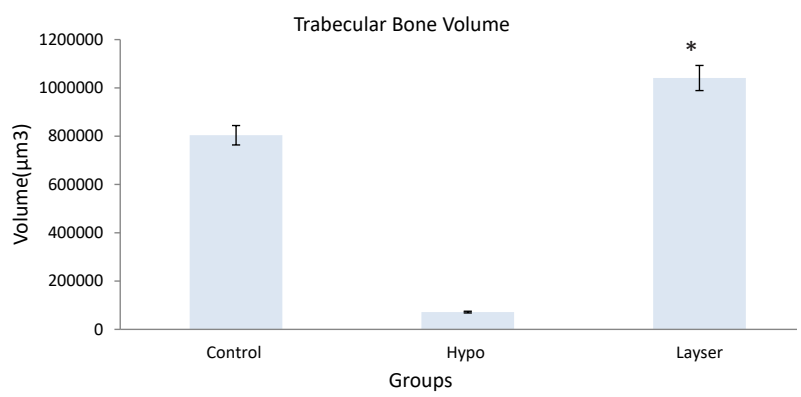

Figure 5. Mean \pm SEM of Trabecular Bone Volume in Different Groups. * shows the significant difference between Hypo group and the other groups $(* P<0.05, * * P<0.01)$.

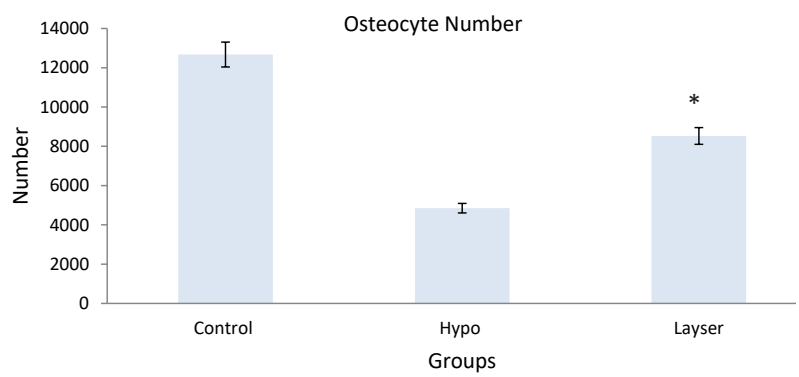

Figure 6. Mean \pm SEM of Number of Osteocytes in Different Groups. * indicates the significant difference between Hypo group and the other groups $\left(* P<0.05,{ }^{* *} P<0.01\right)$.

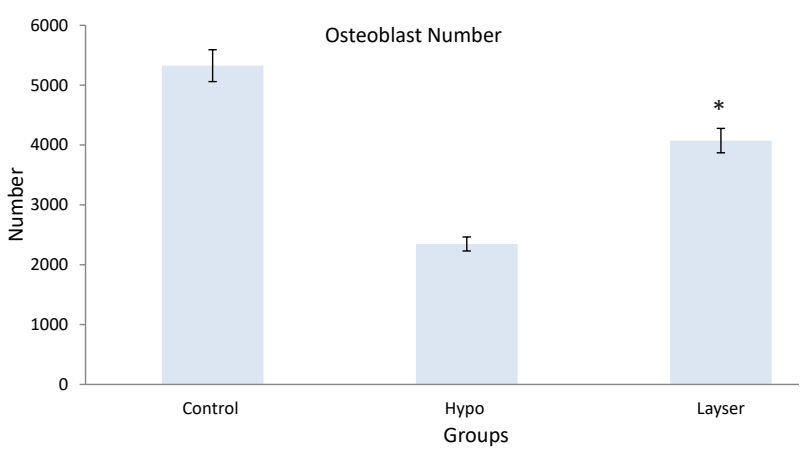

Figure 7. Mean \pm SEM of Number of Osteoblast in Different Groups. * indicates the significant difference between Hypo group and the other groups $\left({ }^{*} P<0.05\right)$. 


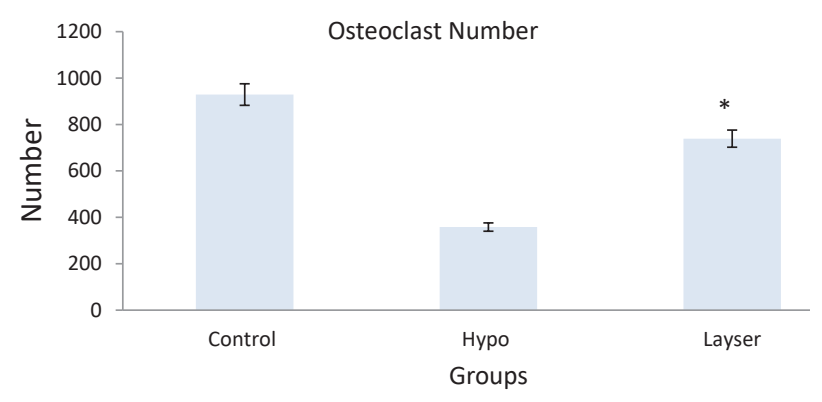

Figure 8. Mean \pm SEM of Osteoclast in Different Groups. * shows the significant difference between Hypo group and the other groups $(* P<0.05, * * P<0.01)$.

number of osteoclasts of the hypothyroid and laser groups $(P<0.05)$.

\section{Discussion}

As a matter of fact, hypothyroidism reduced the biomechanical parameters including bending stiffness (high force load), and energy absorption, as well as the histological parameters including number of bone cells and trabecular bone volume. Disorders of bone regeneration are some of the problems of people with hypothyroidism. Hypothyroidism is one of the most common endocrine diseases and has been associated with bone metabolism abnormalities such as delayed bone regeneration, increased fracture risk, and delayed fracture repair. ${ }^{17,18}$ Reduced thyroid hormones or their receptors causes a variety of disorders in the body metabolism and disrupts normal bone formation. Generally, hypothyroidism disrupts normal bone formation with bone reabsorption impairment, which impairs bone regeneration, delays intracranial and membranous bone formation and decreases growth. Studies have shown that long-term use of levothyroxine can reduce bone density and increase the risk of osteoporosis, which results in delayed fracture recovery in patients with hypothyroidism and a risk of fracture in the hypothyroidism bone. ${ }^{19-21}$ Recent studies have shown osteoclasts and osteoblasts reduced number to decrease bone reabsorption and bone formation in hypothyroid rat model. ${ }^{22}$ The results of this study confirm those of previous studies in that hypothyroidism has been shown to reduce the number of bone cells and also delay the repair of fractures following the reduction of trabecular bone formation. Considering the disturbances that result from hypothyroidism in the skeletal system, treating bone defects caused by trauma, tumor removal, and other diseases in hypothyroidism patients is a major challenge. ${ }^{23}$ We evaluated the fracture healing in rats with hypothyroidism following LLL radiation treatment. The results of this study also showed that LLL improves biomechanical parameters including high-energy stress absorption, as well as histological parameters including increased volume of trabecular bone in rats in low-grade laser therapy group. In various studies, the effects of low-energy laser radiation on various tissues have been reported over the last decade, including: Laser effects on bone repair and reconstruction. Studies have shown that LLL radiation can have stimulatory effects on tissue. Studies have shown that laser radiation can absorb light by mitochondrial chains, which increases mitosis and proliferation of cells. Osteogenic effects of pulsed LLL have also been reported on bone marrow proliferation in vivo and in vitro, and on bone defect and its repair in healthy animals. ${ }^{22,23}$ Medalha et al compared the effects of LLL and pulsed ultrasound on bone repair in partial tibial bone defects. Histological studies show that the number of osteoblasts cells was dramatically increased in ultrasound groups compared with the control group, as well as the reabsorption areas and the number of osteoclasts. Their conclusion was that pulsed ultrasound accelerates bone resorption through absorption and low

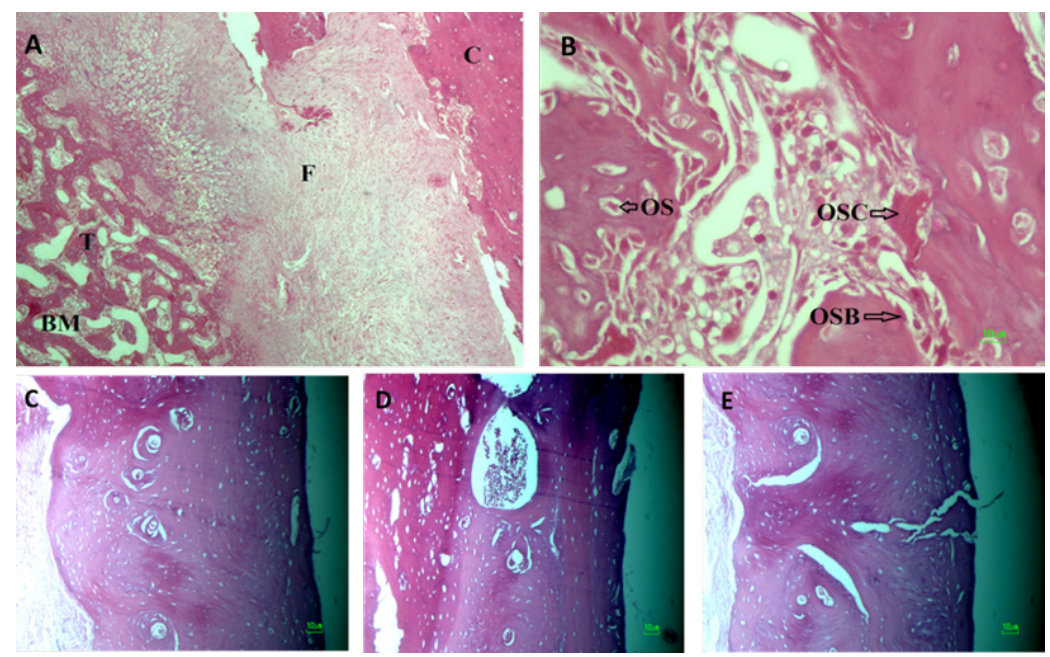

Figure 9. Micrograph of the Bone Defect Stained With H\& E. (A, B) Trabecular Bone (T), Bone Marrow (BM), Fibrous Tissue (F), Cortical Bone (C); (C) Control; (D) Hypo; (E). Hypo + Laser. 
bone laser recovery through bone formation. ${ }^{24}$ Sella et al used a continuous LLL, with a wavelength of $808 \mathrm{~nm}$, and concluded that bone formation rate in the experimental group was dramatically higher in comparison with the control group. These results were consistent with the results of our study. ${ }^{25}$ Nicola et al used a LLL (660 nm) to repair bone defect in rat females. After analyzing the histological findings, it was concluded that LLL propagates all types of bone cells, including osteoblasts and osteoclasts. These results are related to the effects of LLL stimulation on cell proliferation and bone tissue metabolism. ${ }^{26}$ Mostafavinia et al in 2015 examined the effect of LLL radiation with two doses on fracture healing in healthy rats. ${ }^{27}$ In that study, they used a LLL with a wavelength of $890 \mathrm{~nm}$ and a density of 1.5 and $0.972 \mathrm{~J} /$ $\mathrm{cm}^{2}$. The results of that study proved that, using laser radiation, the bone biomechanical parameters including bending stiffness, maximum force, energy absorption, and maximum stress increased significantly compared with control groups. By examining both doses, they concluded that LLL with a density of $1.5 \mathrm{~J} / \mathrm{cm}^{2}$ had a greater effect on elastic stiffness than a LLL with a density of $0.972 \mathrm{~J} /$ $\mathrm{cm}^{2}$. Histologic results in this study showed that laser radiation increased the number of osteoblasts, trabecular bone volume and fibrosis. Our results confirmed those of previous studies in that laser with a density of $1.5 \mathrm{~J} /$ $\mathrm{cm}^{2}$ increased the biomechanical parameters, laser alone could increase the maximum force, maximum stress, and energy absorbed in comparison with the hypothyroid group. ${ }^{27}$ Our histologic results confirmed those of previous studies in that laser increased the trabecula bone relative to the hypothyroid group. Also, there was a significant difference in the volume of bone marrow in the laser group with other group. In this study, it was shown that the amount of bone marrow in the laser group was higher than that of the Hypo group. These results can indicate that the bone formation rate with laser radiation increased more than that in the Hypo group. The results of our research also showed that using LLL radiation can improve biomechanical and histological parameters compared to other groups. So using LLL radiation can increase the osteoblasts, osteoclasts and osteocytes numbers. Also, the new bone formation in the bone defect area in comparison with other groups was significantly different. As noted above, biomechanical results also showed that using LLL can further improve its parameters.

\section{Conclusion}

Hypothyroidism affects the repair of partial fractures in the bone. As osteoblast and osteoclast cells decrease, bone formation and repair are reduced and delayed. Due to the effect of osteoblasts on the process of differentiation of osteoclasts, it is expected that by increasing the number of osteoblast cells, it may increase the differentiation of osteoclast precursor cells to adult osteoclast. However,
LLL significantly increased maximum stress and maximum force, energy and elasticity in the partial bone defect, as well as increased trabecular tissue volume, number of osteoblasts and osteoclasts.

\section{Conflict of Interests}

None.

\section{Ethical Considerations}

Not applicable.

\section{Acknowledgements}

The present article was extracted from a thesis written by Niloofar Sefati for taking Master's degree (Grant No. 1395.52.348). The authors are thankful to the Laser Application in Medical Sciences Research Center of Shahid Beheshti University of Medical Sciences, Tehran, Iran.

\section{References}

1. SefatiN, Norouzian M, Abbaszadeh HA, et al. Effects of bone marrow mesenchymal stem cells-conditioned medium on tibial partial osteotomy model of fracture healing in hypothyroidism rats. Iran Biomed J. 2018;22(2):90-98.

2. Abbaszadeh HA, Tiraihi T, Delshad A, et al. Differentiation of neurosphere-derived rat neural stem cells into oligodendrocyte-like cells by repressing PDGF- $\alpha$ and Olig2 with triiodothyronine. Tissue Cell. 2014;46(6):462-9.

3. Abbaszadeh HA, Tiraihi T, Delshad AR, Saghedi Zadeh M, Taheri T. Bone marrow stromal cell transdifferentiation into oligodendrocyte-like cells using triiodothyronine as a inducer with expression of platelet-derived growth factor alpha as a maturity marker. Iran Biomed J. 2013;17(2):6270 .

4. Vestergaard P, Mosekilde L. Fractures in patients with hyperthyroidism and hypothyroidism: a nationwide followup study in 16,249 patients. Thyroid. 2002;12(5):411-419. doi:10.1089/105072502760043503

5. Gaitonde DY, Rowley KD, Sweeney LB. Hypothyroidism: an update. Am Fam Physician. 2012;86(3):244-251.

6. Karimifar M, Esmaili F, Salari A, Kachuei A, Faragzadegan Z, Karimifar M. Effects of Levothyroxine and thyroid stimulating hormone on bone loss in patients with primary hypothyroidism. J Res Pharm Pract. 2014;3(3):8387. doi:10.4103/2279-042x.141099

7. Garavello I, Baranauskas V, da Cruz-Hofling MA. The effects of low laser irradiation on angiogenesis in injured rat tibiae. Histol Histopathol. 2004;19(1):43-48. doi:10.14670/hh-19.43

8. Mohsenifar Z, Fridoni M, Ghatrehsamani M, et al. Evaluation of the effects of pulsed wave LLLT on tibial diaphysis in two rat models of experimental osteoporosis, as examined by stereological and real-time PCR gene expression analyses. Lasers Med Sci. 2016;31(4):721-732. doi:10.1007/s10103-016-1916-9

9. Abbaszadeh HA, Peyvandi AA, Sadeghi Y, et al. Er:YAG Laser and Cyclosporin A Effect on Cell Cycle Regulation of Human Gingival Fibroblast Cells. J Lasers Med Sci. 2017;8(3):143-149. doi:10.15171/jlms.2017.26 
10. Bolognani L, Volpi N. Low power laser enzymology: Reactivation of myosin ATPase by GaAs an HeNe lasers. In: Passarella S, Quagliariello E, eds. Basic and Applied Research in Photobiology and Photomedicine. Bari, Italy: University of Bari; 1991:315-38.

11. Son Y, Cheong YK, Kim NH, Chung HT, Kang DG, Pae HO. Mitogen-Activated Protein Kinases and Reactive Oxygen Species: How Can ROS Activate MAPK Pathways? J Signal Transduct. 2011;2011:792639. doi:10.1155/2011/792639

12. Arakaki N, Yamashita A, Niimi S, Yamazaki T. Involvement of reactive oxygen species in osteoblastic differentiation of MC3T3-E1 cells accompanied by mitochondrial morphological dynamics. Biomed Res. 2013;34(3):161-166.

13. Amini A, Pouriran R, Abdollahifar MA, et al. Stereological and molecular studies on the combined effects of photobiomodulation and human bone marrow mesenchymal stem cell conditioned medium on wound healing in diabetic rats. $J$ Photochem Photobiol $B$. 2018;182:42-51. doi: 10.1016/j.jphotobiol.2018.03.010

14. Fernandes KR, Ribeiro DA, Rodrigues NC, et al. Effects of low-level laser therapy on the expression of osteogenic genes related in the initial stages of bone defects in rats. J Biomed Opt. 2013;18(3):038002. doi:10.1117/1. jbo.18.3.038002

15. Abbaszadeh HA, Tiraihi T, Sadeghi Y, et al. Decrease in cavity size and oligodendrocyte cell death using neurosphere-derived oligodendrocyte-like cells in spinal cord contusion model. Iran Biomed J. 2017 Oct 15.

16. Noorafshan A, Abdollahifar MA, Asadi-Golshan R, Rashidian-Rashidabadi A, Karbalay-Doust S. Curcumin and sertraline prevent the reduction of the number of neurons and glial cells and the volume of rats' medial prefrontal cortex induced by stress. Acta Neurobiol Exp (Wars). 2014;74(1):44-53.

17. Ranganath SH, Levy O, Inamdar MS, Karp JM. Harnessing the mesenchymal stem cell secretome for the treatment of cardiovascular disease. Cell Stem Cell. 2012;10(3):244-258. doi:10.1016/j.stem.2012.02.005

18. Fadaei Fathabady F, Norouzian M, Azizi F. Effect of hypothyroidism on bone repair in mature female rats. Int J Endocrinol Metab. 2005;3(3):126-9.

19. Tsourdi E, Rijntjes E, Kohrle J, Hofbauer LC, Rauner M. Hyperthyroidism and Hypothyroidism in Male Mice and Their Effects on Bone Mass, Bone Turnover, and the
Wnt Inhibitors Sclerostin and Dickkopf-1. Endocrinology. 2015;156(10):3517-3527. doi:10.1210/en.2015-1073

20. Paraguassu GM, Xavier FC, Cangussu MC, et al. Effect of laser phototherapy (lambda660 $\mathrm{nm}$ ) on type I and III collagen expression during wound healing in hypothyroid rats: an immunohistochemical study in a rodent model. Photomed Laser Surg. 2014;32(5):281-288. doi:10.1089/ pho.2013.3604

21. Chang W, Kim R, Park SI, et al. Enhanced Healing of Rat Calvarial Bone Defects with Hypoxic Conditioned Medium from Mesenchymal Stem Cells through Increased Endogenous Stem Cell Migration via Regulation of ICAM1 Targeted-microRNA-221. Mol Cells. 2015;38(7):643-650. doi:10.14348/molcells.2015.0050

22. Ogata K, Katagiri W, Osugi M, et al. Evaluation of the therapeutic effects of conditioned media from mesenchymal stem cells in a rat bisphosphonate-related osteonecrosis of the jaw-like model. Bone. 2015;74:95-105. doi:10.1016/j.bone.2015.01.011

23. Barbosa D, Villaverde AG, LoschiavoArisawa EA, de Souza RA. Laser therapy in bone repair in rats: analysis of bone optical density. Acta Ortop Bras. 2014;22(2):71-74. doi:10.1590/1413-78522014220200438

24. Medalha CC, Amorim BO, Ferreira JM, et al. Comparison of the effects of electrical field stimulation and low-level laser therapy on bone loss in spinal cord-injured rats. Photomed Laser Surg. 2010;28(5):669-674. doi:10.1089/ pho.2009.2691

25. Sella VR, do Bomfim FR, Machado PC, da Silva Morsoleto MJ, Chohfi M, Plapler H. Effect of low-level laser therapy on bone repair: a randomized controlled experimental study. Lasers Med Sci. 2015;30(3):1061-1068. doi:10.1007/ s10103-015-1710-0

26. Nicola RA, Jorgetti V, Rigau J, Pacheco MT, dos Reis LM, Zangaro RA. Effect of low-power GaAlAs laser (660 nm) on bone structure and cell activity: an experimental animal study. Lasers Med Sci. 2003;18(2):89-94. doi:10.1007/ s10103-003-0260-z

27. Mostafavinia A, Masteri Farahani R, Abbasian M, et al. Effect of Pulsed Wave Low-Level Laser Therapy on Tibial Complete Osteotomy Model of Fracture Healing With an Intramedullary Fixation. Iran Red Crescent Med J. 2015;17(12):e32076. doi:10.5812/ircmj.32076 